\title{
EXTENSIONS AND THE WEAK CALKIN ALGEBRA OF READ'S BANACH SPACE ADMITTING DISCONTINUOUS DERIVATIONS
}

\author{
NIELS JAKOB LAUSTSEN AND RICHARD SKILLICORN
}

In memoriam: Charles J. Read (1958-2015)

\begin{abstract}
Read produced the first example of a Banach space $E_{\mathrm{R}}$ such that the associated Banach algebra $\mathscr{B}\left(E_{\mathrm{R}}\right)$ of bounded operators admits a discontinuous derivation (J. London Math. Soc. 1989). We generalize Read's main theorem about $\mathscr{B}\left(E_{\mathrm{R}}\right)$ from which he deduced this conclusion, as well as the key technical lemmas that his proof relied on, by constructing a strongly split-exact sequence

$$
\{0\} \longrightarrow \mathscr{W}\left(E_{\mathrm{R}}\right) \longrightarrow \mathscr{B}\left(E_{\mathrm{R}}\right) \rightleftarrows \ell_{2}^{\sim} \longrightarrow\{0\}
$$

where $\mathscr{W}\left(E_{\mathrm{R}}\right)$ denotes the ideal of weakly compact operators on $E_{\mathrm{R}}$, while $\ell_{2}^{\sim}$ is the unitization of the Hilbert space $\ell_{2}$, endowed with the zero product.
\end{abstract}

To appear in Studia Mathematica.

\section{INTRODUCTION AND STATEMENT OF THE MAIN RESUlt}

In 1989, Read [7] published the construction of a remarkable Banach space that we shall denote by $E_{\mathrm{R}}$. Read's purpose was to produce an example of a discontinuous derivation from the Banach algebra $\mathscr{B}(E)$ of bounded operators on a Banach space $E$, thus answering an open question in automatic continuity theory going back at least to [4], in which Johnson had shown that, for each Banach space $E$ that is isomorphic to its Cartesian square $E \oplus E$ (or, more generally, such that $E$ has a 'continued bisection'), every homomorphism from $\mathscr{B}(E)$ into a Banach algebra is continuous. A general result states that the continuity of all homomorphisms from a Banach algebra implies the continuity of all derivations from it; see, e.g., [2, Theorem 2.7.5(i)]. Hence Read's result implies that Johnson's theorem does not extend to all Banach spaces, and it was the first example of a Banach space $E$ for which $\mathscr{B}(E)$ admits a discontinuous homomorphism.

Read's strategy was to establish the following theorem, from which the existence of a discontinuous derivation from $\mathscr{B}\left(E_{\mathrm{R}}\right)$ into a one-dimensional Banach $\mathscr{B}\left(E_{\mathrm{R}}\right)$-bimodule follows by standard methods, as detailed in [7, Theorem 1].

Theorem 1.1 (Read). There exists a Banach space $E_{\mathrm{R}}$ such that the Banach algebra $\mathscr{B}\left(E_{\mathrm{R}}\right)$ contains a closed ideal $\mathscr{I}$ of codimension one, but the closed linear span of its

2010 Mathematics Subject Classification. Primary 46H10, 46M18, 47L10; Secondary 16 S70.

Key words and phrases. Bounded operator; Read's Banach space; Banach algebra; short-exact sequence; strong splitting; discontinuous derivation. 
products,

$$
\mathscr{I}^{2}=\overline{\operatorname{span}}\{S T: S, T \in \mathscr{I}\},
$$

has infinite codimension in $\mathscr{B}\left(E_{\mathrm{R}}\right)$. More precisely,

(i) $\mathscr{I}$ contains the ideal $\mathscr{W}\left(E_{\mathrm{R}}\right)$ of weakly compact operators on $E_{\mathrm{R}}$;

(ii) $\mathscr{W}\left(E_{\mathrm{R}}\right)$ has infinite codimension in $\mathscr{B}\left(E_{\mathrm{R}}\right)$;

(iii) $\mathscr{I}^{2} \subseteq \mathscr{W}\left(E_{\mathrm{R}}\right)$.

This theorem will be an immediate consequence of our main result, which will also incorporate and strengthen the key technical lemmas that Read used to establish it, as we shall explain below, once we have stated our main result precisely. It involves the following notation. Endow the separable, infinite-dimensional Hilbert space $\ell_{2}$ with the zero product, and denote its unitization by $\ell_{2}^{\sim}$, so that $\ell_{2}^{\sim}=\ell_{2} \oplus \mathbb{K} 1$ as a vector space (where $\mathbb{K}$ denotes the scalar field, either $\mathbb{R}$ or $\mathbb{C}$, and 1 is the formal identity that we adjoin), and the product and norm on $\ell_{2}^{\sim}$ are given by

$$
(\xi+\lambda 1)(\eta+\mu 1)=\lambda \eta+\mu \xi+\lambda \mu 1 \quad \text { and } \quad\|\xi+\lambda 1\|=\|\xi\|+|\lambda| \quad\left(\xi, \eta \in \ell_{2}, \lambda, \mu \in \mathbb{K}\right) .
$$

Theorem 1.2. There exists a continuous, surjective homomorphism $\psi$ from the Banach algebra $\mathscr{B}\left(E_{\mathrm{R}}\right)$ onto $\ell_{2}^{\sim}$ with $\operatorname{ker} \psi=\mathscr{W}\left(E_{\mathrm{R}}\right)$ such that the short-exact sequence

$$
\{0\} \longrightarrow \mathscr{W}\left(E_{\mathrm{R}}\right) \longrightarrow \mathscr{B}\left(E_{\mathrm{R}}\right) \stackrel{\psi}{\longrightarrow} \ell_{2}^{\sim} \longrightarrow\{0\}
$$

splits strongly, in the sense that there is a continuous homomorphism from $\ell_{2}^{\sim}$ into $\mathscr{B}\left(E_{\mathrm{R}}\right)$ which is a right inverse of $\psi$.

Taking $\mathscr{I}$ to be the preimage under $\psi$ of the codimension-one ideal $\ell_{2}$ of $\ell_{2}^{\sim}$, we see that clauses (i)-(iii) of Theorem 1.1 are satisfied, so that Theorem 1.1 follows from Theorem 1.2, as claimed above. To explain how Theorem 1.2 incorporates and strengthens the key technical lemmas in Read's construction, let us first describe the latter in more detail.

After defining the Banach space $E_{\mathrm{R}}$, Read begins his study of it by showing that the quotient $E_{\mathrm{R}}^{* *} / E_{\mathrm{R}}$ is isomorphic to a Hilbert space $H$, and he then identifies a particular orthonormal basis $\left(a_{n}\right)_{n \in \mathbb{N}}$ for this Hilbert space (see [7, equation (3.6.5) and Lemma 3.7]). Given an operator $T \in \mathscr{B}\left(E_{\mathrm{R}}\right)$, its bidual $T^{* *}$ induces an operator $\Theta_{0}(T)$ on $E_{\mathrm{R}}^{* *} / E_{\mathrm{R}}$, and hence on $H$, by a standard construction, as detailed in the diagram (2.1) below. Read's main technical achievement is [7, Lemma 4.1], which states that when $\Theta_{0}(T)$ is considered as an operator on $H$, its matrix $M$ with respect to the orthonormal basis $\left(a_{n}\right)_{n \in \mathbb{N}}$ has a very special form, namely

$$
M=\left(\begin{array}{cccccccccc}
\lambda & 0 & 0 & \ldots & & & & & & \\
\mu_{1} & \lambda & 0 & 0 & \ldots & & & & & \\
\mu_{1} & 0 & \lambda & 0 & 0 & \ldots & & & & \\
\mu_{2} & 0 & 0 & \lambda & 0 & 0 & \ldots & & & \\
\mu_{2} & 0 & 0 & 0 & \lambda & 0 & 0 & \ldots & & \\
\mu_{3} & 0 & 0 & 0 & 0 & \lambda & 0 & 0 & \ldots & \\
\mu_{3} & 0 & 0 & 0 & 0 & 0 & \lambda & 0 & 0 & \ldots \\
\vdots & \vdots & \vdots & \vdots & \vdots & \vdots & & \ddots & &
\end{array}\right)
$$


for some scalars $\lambda$ and $\mu_{1}, \mu_{2}, \mu_{3}, \ldots$ (Note that (1.1) corrects a typo in [7, equation (4.1.1)]: the first entry of the fifth row of $M$ should be $\mu_{2}$, not $\mu_{3}$, as [7, Lemma 4.1(c)] shows.) Finally, in [7, Lemma 4.2], Read establishes a partial converse to this result by showing that, in the case where only finitely many of the scalars $\mu_{1}, \mu_{2}, \mu_{3}, \ldots$ are non-zero, $(1.1)$ arises as the matrix of $\Theta_{0}(T)$ for some operator $T \in \mathscr{B}\left(E_{\mathrm{R}}\right)$.

The connection between these results and Theorem 1.2 goes via the following observation. The first column of the matrix (1.1) is the image of the first basis vector under the operator $\Theta_{0}(T)$, so that the sequence $\left(\mu_{i}\right)_{i \in \mathbb{N}}$ belongs to $\ell_{2}$. Hence we can define a mapping $\psi: \mathscr{B}\left(E_{\mathrm{R}}\right) \rightarrow \ell_{2}^{\sim}$ by $\psi(T)=\left(\mu_{i}\right)_{i \in \mathbb{N}}+\lambda 1$, where $\lambda$ and $\mu_{1}, \mu_{2}, \ldots$ are the scalars determined by $\Theta_{0}(T)$ via (1.1). We shall show that this mapping $\psi$ is the surjective homomorphism whose existence is stated in Theorem 1.2, which therefore strengthens Read's technical lemmas in two ways. First, the surjectivity of $\psi$ means that all possible matrices of the form (1.1) arise as the matrix of $\Theta_{0}(T)$ for some operator $T \in \mathscr{B}\left(E_{\mathrm{R}}\right)$, not just those whose first column vanishes eventually. Second, we can choose a preimage $T$ under $\psi$ of the element $\left(\mu_{i}\right)_{i \in \mathbb{N}}+\lambda 1 \in \ell_{2}^{\sim}$ in such a way that the corresponding mapping is a bounded homomorphism.

Our motivation for proving Theorem 1.2 is that it has enabled us to show that:

- the Banach algebra $\mathscr{B}\left(E_{\mathrm{R}}\right)$ has a singular extension which splits algebraically, but it is not admissible, and so does not split strongly;

- the homological bidimension of $\mathscr{B}\left(E_{\mathrm{R}}\right)$ is at least two.

The first of these results solves a natural problem left open in Bade, Dales, and Lykova's comprehensive study [1] of splittings of extensions of Banach algebras, while the second answers a question originating in Helemskii's seminar at Moscow State University. For details of any unexplained terminology and how to deduce these results from Theorem 1.2, we refer to [6].

Note added in proof. After the completion of [6], in joint work with Kania [5], we have succeeded in constructing a singular extension of $\mathscr{B}\left(E_{\mathrm{R}}\right)$ which is admissible and splits algebraically, but does not split strongly, thus complementing the first of the two results mentioned above. Once again, Theorem 1.2 plays a key role in the proof of this result.

To conclude this Introduction, we would like to express the hope that, by elucidating the structure of the Banach algebra $\mathscr{B}\left(E_{\mathrm{R}}\right)$, Theorem 1.2 will spark new interest in and lead to further applications of this remarkable creation of Read's.

\section{ReAD's BANACH SPACE $E_{\mathrm{R}}$ AND The PROOF OF THEOREM 1.2}

We begin this section with some general conventions and results, followed by an overview of Read's construction of the Banach space $E_{\mathrm{R}}$ and details of the specific elements of Read's work that we shall require in our proof of Theorem 1.2, before we conclude with the proof itself. Our aim is to provide enough detail to make this presentation self-contained, without repeating arguments already given in [7]. An expanded, entirely self-contained version of the proof of Theorem 1.2, incorporating all necessary details of Read's construction, can be found in [8, Chapter 5]. 
We shall generally follow the notation and terminology used in [7]; in some places, however, we add extra details or take a slightly different view from Read's in order to facilitate our proof of Theorem 1.2 and avoid ambiguities.

All results in [7] are stated for complex scalars only. We observe that the proofs carry over verbatim to the real case, so we shall address both cases simultaneously, denoting the scalar field by $\mathbb{K}$, so that either $\mathbb{K}=\mathbb{R}$ or $\mathbb{K}=\mathbb{C}$.

By an operator, we understand a bounded, linear mapping between Banach spaces. For a Banach space $E$, we denote by $E^{*}$ its dual space, and we identify $E$ with its canonical image in the bidual space $E^{* *}$. Let $\pi_{E}: E^{* *} \rightarrow E^{* *} / E$ denote the quotient mapping. Since the restriction to $E$ of the bidual $T^{* *}$ of an operator $T \in \mathscr{B}(E)$ is equal to $T$, it leaves the subspace $E$ invariant, and hence the Fundamental Isomorphism Theorem implies that there is a unique operator $\Theta_{0}(T) \in \mathscr{B}\left(E^{* *} / E\right)$ such that the diagram

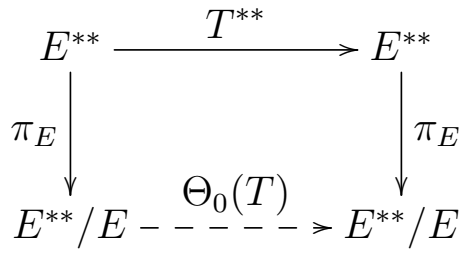

is commutative. Moreover, the mapping $\Theta_{0}: T \mapsto \Theta_{0}(T), \mathscr{B}(E) \rightarrow \mathscr{B}\left(E^{* *} / E\right)$, is a continuous, unital algebra homomorphism of norm at most one, and a standard characterization of the ideal of weakly compact operators shows that $\operatorname{ker} \Theta_{0}=\mathscr{W}(E)$.

Let $\left(A,\|\cdot\|_{A}\right)$ be a Banach space with a normalized, symmetric basis $\left(e_{n}\right)_{n \in \mathbb{N}}$. By passing to an equivalent norm, we may suppose that the basis $\left(e_{n}\right)_{n \in \mathbb{N}}$ is 1-symmetric, that is, for each $n \in \mathbb{N}$, each permutation $\pi$ of $\mathbb{N}$, and all scalars $\lambda_{1}, \ldots, \lambda_{n}, \mu_{1}, \ldots, \mu_{n}$, we have

$$
\left\|\sum_{j=1}^{n} \lambda_{j} \mu_{j} e_{\pi(j)}\right\|_{A} \leqslant \max \left\{\left|\mu_{1}\right|, \ldots,\left|\mu_{n}\right|\right\}\left\|\sum_{j=1}^{n} \lambda_{j} e_{j}\right\|_{A} .
$$

Read [7, Definition 1.2(b)] defines the James-like space $J A$ based on $A$ as the collection of all scalar sequences $x=\left(\lambda_{n}\right)_{n \in \mathbb{N}} \in c_{0}$ such that the quantity

$$
\begin{array}{r}
\|x\|_{J A}=\sup \left\{\left\|\sum_{j=1}^{n}\left(\lambda_{p_{j}}-\lambda_{p_{j+1}}\right)^{2} e_{j}+\lambda_{p_{n+1}}^{2} e_{n+1}\right\|_{A}^{\frac{1}{2}}:\right. \\
\left.n, p_{1}, \ldots, p_{n+1} \in \mathbb{N}, p_{1}<p_{2}<\cdots<p_{n+1}\right\}
\end{array}
$$

is finite, and observes that $\left(J A,\|\cdot\|_{J A}\right)$ is a Banach space.

In [7, Section 2], Read proceeds to show that, in the case where $A$ contains no subspace isomorphic to $c_{0}$, the space $J A$ is quasi-reflexive of order one, so that the canonical image of $J A$ has codimension one in its bidual $(J A)^{* *}$, just like the original James space (which in this approach corresponds to $\left.A=\ell_{1}\right)$. Moreover, the standard unit vector basis $\left(e_{n}\right)_{n \in \mathbb{N}}$ is a shrinking basis for $J A$, so that the sequence $\left(e_{n}^{*}\right)_{n \in \mathbb{N}}$ of coordinate functionals is a basis for the dual space $(J A)^{*}$. Like Read, we use $\left(e_{n}\right)_{n \in \mathbb{N}}$ to denote the basis of both $A$ and $J A$, and 
we identify $(J A)^{* *}$ with $J A \oplus \mathbb{K} \Phi$, where $\Phi$ is the functional on $(J A)^{*}$ given by $\left\langle e_{n}^{*}, \Phi\right\rangle=1$ for each $n \in \mathbb{N}$. We record for later use that $\|\Phi\|_{(J A)^{* *}}=1$ by [7, Lemma 2.2(a)].

Let $\mathbb{N}_{0}=\mathbb{N} \cup\{0\}$ be the set of non-negative integers, and set

$$
\mathbb{I}=\{2\} \cup\left\{i \in \mathbb{N}_{0}: i \equiv 0 \bmod 6, i \equiv 4 \bmod 6 \text {, or } i \equiv 5 \bmod 6\right\} .
$$

For each $i \in \mathbb{I}$, choose a Banach space $\left(B_{i},\|\cdot\|_{B_{i}}\right)$ with a normalized, 1-symmetric basis, which we denote by $\left(e_{n}\right)_{n \in \mathbb{N}}$ (independent of $i$ ), such that no subspace of $B_{i}$ is isomorphic to $c_{0}$, and such that the family $\left(B_{i}\right)_{i \in \mathbb{I}}$ is incomparable, in the sense that, for each $i \in \mathbb{I}$ and each $\varepsilon>0$, there exists $n \in \mathbb{N}$ with

$$
\left\|\sigma_{n}\right\|_{B_{i}} \leqslant \varepsilon \cdot \inf \left\{\left\|\sigma_{n}\right\|_{B_{j}}: j \in \mathbb{I} \backslash\{i\}\right\},
$$

where $\sigma_{n}=\sum_{k=1}^{n} e_{k}$; Read explains in the note following [7, Definition 3.2] that such a family exists. For $i \in \mathbb{N} \backslash \mathbb{I}$, set

$$
B_{i}= \begin{cases}B_{0} & \text { if } i \equiv 1 \bmod 6 \\ B_{2} & \text { if } i \equiv 2 \bmod 6 \text { or } i \equiv 3 \bmod 6,\end{cases}
$$

so that $B_{i}$ is now defined for each $i \in \mathbb{N}_{0}$, and we have a sequence $\left(J B_{i}\right)_{i \in \mathbb{N}_{0}}$ of Jameslike Banach spaces, whose $\ell_{2}$-direct sum $Y=\left(\oplus_{i=0}^{\infty} J B_{i}\right)_{\ell_{2}}$ is at the heart of Read's construction. By standard duality and the quasi-reflexivity of the spaces $J B_{i}$, we have the following identifications:

$$
Y^{*}=\left(\bigoplus_{i=0}^{\infty}\left(J B_{i}\right)^{*}\right)_{\ell_{2}} \quad \text { and } \quad Y^{* *}=\left(\bigoplus_{i=0}^{\infty}\left(J B_{i}\right)^{* *}\right)_{\ell_{2}}=\left(\bigoplus_{i=0}^{\infty} J B_{i} \oplus \mathbb{K} \Phi\right)_{\ell_{2}} .
$$

Another important piece of notation involves the Hilbert space $\bar{B}=\ell_{2}\left(\mathbb{N}_{0}\right)$ and its standard orthonormal basis $\left(b_{n}\right)_{n \in \mathbb{N}_{0}}$, which Read relabels as follows for positive indices:

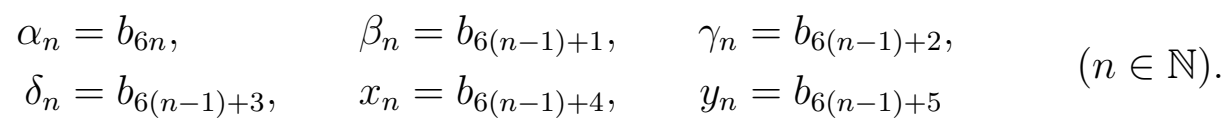

For $n \in \mathbb{N}$ and $\xi=\sum_{i=0}^{\infty} \xi_{i} b_{i} \in \bar{B}$, Read introduces the tensor notation $e_{n} \otimes \xi=\left(\xi_{i} e_{n}\right)_{i=0}^{\infty}$, which defines an element of $Y$ due to the uniform bound $\left\|e_{n}\right\|_{J B_{i}} \leqslant\left\|e_{1}+e_{2}\right\|_{B_{i}} \leqslant 2$, independent of $i \in \mathbb{N}_{0}$. This definition extends by linearity to tensors of the form $x \otimes \xi$ for $x \in c_{00}$. By assigning special symbols to the following linear combinations of the basis vectors $(2.5)$,

$$
\begin{array}{ll}
\alpha_{n}^{\prime}=\alpha_{n}-\left(x_{n}-y_{n}\right), & \beta_{n}^{\prime}=\beta_{n}-\left(x_{n}+y_{n}\right), \\
\gamma_{n}^{\prime}=\gamma_{n}-\left(x_{n}+y_{n}\right), & \delta_{n}^{\prime}=\delta_{n}-\left(\frac{b_{0}}{2^{n}}-x_{n}+y_{n}\right)
\end{array} \quad(n \in \mathbb{N}),
$$

we can now define Read's space $E_{\mathrm{R}}$ as follows:

$$
\begin{aligned}
S & =\left\{\alpha_{n}^{\prime}, \beta_{n}^{\prime}, \gamma_{n}^{\prime}, \delta_{n}^{\prime}: n \in \mathbb{N}\right\}, & V & =\overline{\operatorname{span}} S \subseteq \bar{B}, \\
N & =\overline{\operatorname{span}}\left\{e_{n} \otimes s: n \in \mathbb{N}, s \in S\right\} \subseteq Y, & E_{\mathrm{R}} & =Y / N .
\end{aligned}
$$


Note. Our definition of $\beta_{n}^{\prime}$ above corrects a typo in [7, Definition 3.4(b)], where the sign of $y_{n}$ is wrong, as one can see by comparing it with the second line of the displayed equations at the bottom of [7, p. 313] and the seventh displayed equation of [7, p. 319].

As we stated in the Introduction, the first two steps in Read's analysis of the space $E_{\mathrm{R}}$ consist of showing that the quotient $E_{\mathrm{R}}^{* *} / E_{\mathrm{R}}$ is isomorphic to a Hilbert space and identifying an orthonormal basis for it. Read, however, does not obtain an explicit formula for this isomorphism. Such a formula will be required in our proof of Theorem 1.2, so we shall now recast Read's arguments in a form that will produce an explicit isomorphism $U$ between the closed subspace

$$
H=\overline{\operatorname{span}}\left\{b_{0}, x_{n}, y_{n}: n \in \mathbb{N}\right\}
$$

of $\bar{B}$ and $E_{\mathrm{R}}^{* *} / E_{\mathrm{R}}$.

To this end, we observe that the proof of [7, Lemma 3.7] shows that the restriction to $H$ of the quotient mapping $Q_{V}: \bar{B} \rightarrow \bar{B} / V$ is surjective and bounded below by $\frac{1}{15}$; that is, $\left.Q_{V}\right|_{H}$ is an isomorphism whose inverse has norm at most 15. (Read denotes this mapping by $\alpha$; we prefer $\left.Q_{V}\right|_{H}$ as it is more descriptive and avoids any possible confusion with the basis vectors $\alpha_{n}$.)

Set $U_{0}=\pi_{E_{\mathrm{R}}} Q_{N}^{* *} R_{0}: \bar{B} \rightarrow E_{\mathrm{R}}^{* *} / E_{\mathrm{R}}$, where $\pi_{E_{\mathrm{R}}}: E_{\mathrm{R}}^{* *} \rightarrow E_{\mathrm{R}}^{* *} / E_{\mathrm{R}}$ and $Q_{N}: Y \rightarrow E_{\mathrm{R}}$ are the quotient mappings, and $R_{0}: \bar{B} \rightarrow Y^{* *}$ is the linear isometry given by

$$
R_{0} \xi=\Phi \otimes \xi=\left(\xi_{i} \Phi\right)_{i=0}^{\infty} \quad\left(\xi=\sum_{i=0}^{\infty} \xi_{i} b_{i} \in \bar{B}\right)
$$

using the natural extension to $Y^{* *}$ of Read's tensor notation for $Y$ introduced above. We claim that $U_{0}$ is surjective with $\operatorname{ker} U_{0}=V$. To establish this claim, we first note that $\operatorname{ker} Q_{N}^{* *}=N^{\circ \circ}$ (the bipolar of $N$ ) by a standard duality result, and therefore

$$
\operatorname{ker}\left(\pi_{E_{\mathrm{R}}} Q_{N}^{* *}\right)=Y+N^{\circ \circ}
$$

Equation (2.4) shows that $Y^{* *}=Y+R_{0}[\bar{B}]$. Combining this with the surjectivity of $\pi_{E_{\mathrm{R}}} Q_{N}^{* *}$ and (2.9), we obtain $E_{\mathrm{R}}^{* *} / E_{\mathrm{R}}=\pi_{E_{\mathrm{R}}} Q_{N}^{* *}\left[Y^{* *}\right]=U_{0}[\bar{B}]$, so that $U_{0}$ is indeed surjective.

Next, to determine the kernel of $U_{0}$, we require Read's observation [7, equation (3.6.3)] that each element $x^{* *}$ of $Y^{* *}$ can be expressed uniquely as $x^{* *}=\Phi \otimes \eta_{0}+\sum_{i=1}^{\infty} e_{i} \otimes \eta_{i}$, where $\eta_{i} \in \bar{B}$ for each $i \in \mathbb{N}_{0}$. Using this notation, Read [7, Lemma 3.6.4] shows that

$$
N^{\circ \circ}=\left\{x^{* *}: \eta_{i} \in V \text { for each } i \in \mathbb{N}_{0}\right\} \text {, }
$$

which together with (2.9) immediately implies that $V \subseteq \operatorname{ker} U_{0}$.

Conversely, suppose that $\xi \in \operatorname{ker} U_{0}$. Then $\Phi \otimes \xi \in \operatorname{ker}\left(\pi_{E_{\mathrm{R}}} Q_{N}^{* *}\right)$, so by (2.9), we can find $y=\sum_{i=1}^{\infty} e_{i} \otimes \eta_{i} \in Y$ such that $\Phi \otimes \xi-y \in N^{\circ \circ}$, and hence $\xi \in V$ by (2.10) (as well as $\eta_{i} \in V$ for each $i \in \mathbb{N}$, but we do not need this information). This proves our claim. 
By the Fundamental Isomorphism Theorem, there is a unique isomorphism $\widehat{U}_{0}$ of $\bar{B} / V$ onto $E_{\mathrm{R}}^{* *} / E_{\mathrm{R}}$ such that $U_{0}=\widehat{U}_{0} Q_{V}$, and hence we have a commutative diagram

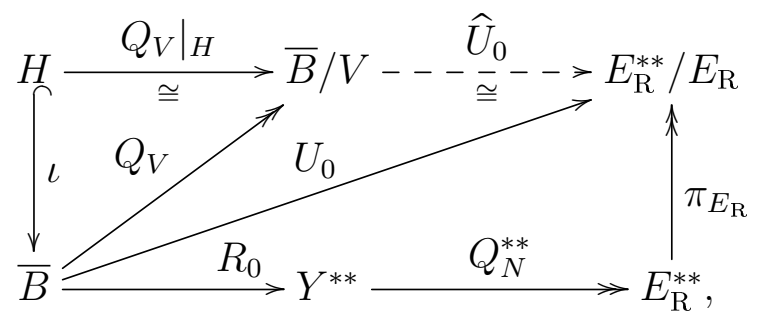

where $\iota: H \rightarrow \bar{B}$ denotes the natural inclusion.

This diagram shows in particular that the restriction $U$ of the operator $U_{0}$ to the subspace $H$ is an isomorphism onto $E_{\mathrm{R}}^{* *} / E_{\mathrm{R}}$, and so it induces a continuous algebra isomorphism Ad $U: T \mapsto U^{-1} T U$ of the Banach algebra $\mathscr{B}\left(E_{\mathrm{R}}^{* *} / E_{\mathrm{R}}\right)$ onto $\mathscr{B}(H)$. Using this notation, we can rephrase [7, Lemma 4.1] as follows: for each $T \in \mathscr{B}\left(E_{\mathrm{R}}\right)$, the matrix $M$ of the operator $(\operatorname{Ad} U) \circ \Theta_{0}(T)$ with respect to the orthonormal basis $\left(a_{n}\right)_{n \in \mathbb{N}}=\left(b_{0}, x_{n}, y_{n}\right)_{n \in \mathbb{N}}$ for $H$ is given by (1.1). Alternatively, we can express this identity as

$$
(\operatorname{Ad} U) \circ \Theta_{0}(T)=\lambda I_{H}+\tau_{\xi},
$$

where $I_{H}$ is the identity operator on $H, \xi=\sum_{n=1}^{\infty} \mu_{n}\left(x_{n}+y_{n}\right)$, and $\tau_{\xi}$ is the rank-one operator on $H$ given by $\eta \mapsto\left(\eta \mid b_{0}\right) \xi$, where $\left(\eta \mid b_{0}\right)$ denotes the inner product of $\eta$ and $b_{0}$. We note in passing that the fact that the spaces $\left(B_{i}\right)_{i \in \mathbb{I}}$ are chosen to be incomparable in the sense of (2.2) plays a crucial role in the proof of [7, Lemma 4.1].

We are now ready to prove Theorem 1.2. Our proof refines that of [7, Lemma 4.2], as it is given in [7, p. 320]. For clarity, we shall present a fully self-contained argument.

Proof of Theorem 1.2. For notational convenience, we shall replace the generic Hilbert space $\ell_{2}$ in the statement of Theorem 1.2 with the closed subspace

$$
H_{0}=\overline{\operatorname{span}}\left\{x_{n}+y_{n}: n \in \mathbb{N}\right\}
$$

of the Hilbert space $H$ given by (2.8). The space $H_{0}$ is of course isometrically isomorphic to $\ell_{2}$ via the mapping $\xi \mapsto\left(\frac{1}{\sqrt{2}}\left(\xi \mid x_{n}+y_{n}\right)\right)_{n \in \mathbb{N}}$, so this is really only a change in notation, provided that we endow $H_{0}$ with the zero product. Then, using that the vector $b_{0}$ is orthogonal to $H_{0}$, we see that the mapping $\Upsilon: \xi+\lambda 1 \mapsto \tau_{\xi}+\lambda I_{H}$ is a continuous, unital algebra isomorphism of the unitization $\widetilde{H}_{0}=H_{0} \oplus \mathbb{K} 1$ of $H_{0}$ (defined analogously to that of $\ell_{2}$ given on page 2) onto the closed subalgebra $\mathscr{T}=\left\{\tau_{\xi}+\lambda I_{H}: \xi \in H_{0}, \lambda \in \mathbb{K}\right\}$ of $\mathscr{B}(H)$.

By (2.12), the range of $(\operatorname{Ad} U) \circ \Theta_{0}$ is contained in $\mathscr{T}$, so we may consider the composite continuous algebra homomorphism $\psi$ given by

$$
\psi=\Upsilon^{-1} \circ(\operatorname{Ad} U) \circ \Theta_{0}: \mathscr{B}\left(E_{\mathrm{R}}\right) \rightarrow \widetilde{H}_{0} .
$$

Since $\Upsilon^{-1}$ and $\operatorname{Ad} U$ are both isomorphisms, we see that $\operatorname{ker} \psi=\operatorname{ker} \Theta_{0}=\mathscr{W}\left(E_{\mathrm{R}}\right)$. It remains to construct a continuous algebra homomorphism which is a right inverse of $\psi$.

Let $\xi=\sum_{n=1}^{\infty} \xi_{n}\left(x_{n}+y_{n}\right) \in H_{0}$ and $y=(y(i))_{i=0}^{\infty} \in Y$ be given, where $y(i) \in J B_{i}$ for each $i \in \mathbb{N}_{0}$. By (2.3), we have $J B_{6(n-1)+1}=J B_{0}$ for each $n \in \mathbb{N}$, so that in analogy with 
the tensor notation already introduced, we may define $y(0) \otimes \beta_{n}$ to be the element of $Y$ whose $(6(n-1)+1)^{\text {st }}$ coordinate is $y(0)$, while all other coordinates vanish. Then the series $\sum_{n=1}^{\infty} \xi_{n} y(0) \otimes \beta_{n}$ converges in $Y$, and its sum has norm $\frac{1}{\sqrt{2}}\|\xi\|_{\bar{B}}\|y(0)\|_{J B_{0}}$.

Using (2.3) once more, we see that $J B_{6(n-1)+2}=J B_{6(n-1)+3}=J B_{2}$ for each $n \in \mathbb{N}$, so that we have an absolutely convergent series

$$
\sum_{n=1}^{\infty} \frac{y(6(n-1)+3)}{2^{n}}
$$

in $J B_{2}$, whose sum $y^{\prime}$ has norm at most $\|y\|_{Y}$. As above, let $y^{\prime} \otimes \gamma_{n}$ be the element of $Y$ whose $(6(n-1)+2)^{\text {nd }}$ coordinate is $y^{\prime}$, while all other coordinates vanish. Then the series $\sum_{n=1}^{\infty} \xi_{n} y^{\prime} \otimes \gamma_{n}$ converges in $Y$, and its sum has norm at most $\frac{1}{\sqrt{2}}\|\xi\|_{\bar{B}}\|y\|_{Y}$.

Combining these conclusions, we may define an element of $Y$ by

$$
T_{\xi} y=\sum_{n=1}^{\infty} \xi_{n}\left(y(0) \otimes \beta_{n}+\left(\sum_{m=1}^{\infty} \frac{y(6(m-1)+3)}{2^{m}}\right) \otimes \gamma_{n}\right),
$$

and $\left\|T_{\xi} y\right\|_{Y} \leqslant \sqrt{2}\|\xi\|_{\bar{B}}\|y\|_{Y}$. The mapping $T_{\xi}: y \mapsto T_{\xi} y$ is clearly linear and has norm at most $\sqrt{2}\|\xi\|_{\bar{B}}$, and hence we have a mapping

$$
\rho_{0}: \xi \mapsto T_{\xi}, \quad H_{0} \rightarrow \mathscr{B}(Y)
$$

which is linear and bounded with norm at most $\sqrt{2}$.

To prove that $\rho_{0}$ is multiplicative, we must show that $T_{\eta} T_{\xi}=0$ for each pair $\xi, \eta \in H_{0}$ because $H_{0}$ has the zero product. Write $\xi$ as $\xi=\sum_{n=1}^{\infty} \xi_{n}\left(x_{n}+y_{n}\right)$, and let $y=(y(i))_{i=0}^{\infty} \in Y$ be given. By (2.13), the element $z=T_{\xi} y$ has the form $z=(z(i))_{i=0}^{\infty}$, where

$$
z(i)= \begin{cases}\xi_{n} y(0) & \text { if } i=6(n-1)+1 \text { for some } n \in \mathbb{N} \\ \xi_{n} \sum_{m=1}^{\infty} \frac{y(6(m-1)+3)}{2^{m}} & \text { if } i=6(n-1)+2 \text { for some } n \in \mathbb{N} \\ 0 & \text { otherwise }\end{cases}
$$

Hence $z(0)=0=z(6(m-1)+3)$ for each $m \in \mathbb{N}$, so that another application of $(2.13)$ shows that $0=T_{\eta} z=T_{\eta} T_{\xi} y$, as required.

Next, we shall prove that

$$
T_{\xi}[N] \subseteq N \quad\left(\xi \in H_{0}\right),
$$

where $N$ is the subspace of $Y$ given by (2.7). Since $T_{\xi}$ is bounded and linear, it suffices to show that $T_{\xi}\left(e_{n} \otimes s\right) \in N$ for each $n \in \mathbb{N}$ and $s \in S$. Comparing (2.13) with (2.5), we see that $T_{\xi}\left(e_{n} \otimes \eta\right)=0$ for each $\eta \in\left\{\alpha_{m}, \beta_{m}, \gamma_{m}, x_{m}, y_{m}: m \in \mathbb{N}\right\}$, and therefore $T_{\xi}\left(e_{n} \otimes s\right)=0 \in N$ for each $s \in\left\{\alpha_{m}^{\prime}, \beta_{m}^{\prime}, \gamma_{m}^{\prime}: m \in \mathbb{N}\right\}$ by (2.6). Moreover, for $m \in \mathbb{N}$, we 
have

$$
\begin{aligned}
T_{\xi}\left(e_{n} \otimes \delta_{m}^{\prime}\right) & =T_{\xi}\left(e_{n} \otimes \delta_{m}\right)-\frac{1}{2^{m}} T_{\xi}\left(e_{n} \otimes b_{0}\right)=\sum_{k=1}^{\infty} \xi_{k} \frac{e_{n}}{2^{m}} \otimes \gamma_{k}-\frac{1}{2^{m}} \sum_{k=1}^{\infty} \xi_{k} e_{n} \otimes \beta_{k} \\
& =\frac{1}{2^{m}} \sum_{k=1}^{\infty} \xi_{k} e_{n} \otimes\left(\gamma_{k}-\beta_{k}\right)=\frac{1}{2^{m}} \sum_{k=1}^{\infty} \xi_{k} e_{n} \otimes\left(\gamma_{k}^{\prime}-\beta_{k}^{\prime}\right) \in N
\end{aligned}
$$

which completes the proof of (2.15).

Thus, by the Fundamental Isomorphism Theorem, there is a unique operator $\breve{T}_{\xi} \in \mathscr{B}\left(E_{\mathrm{R}}\right)$ such that the diagram

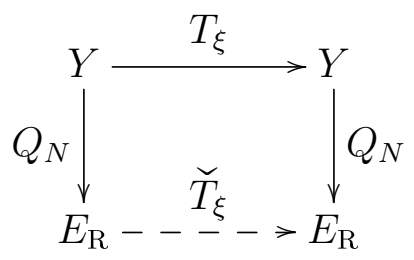

is commutative, and $\left\|\breve{T}_{\xi}\right\|=\left\|Q_{N} T_{\xi}\right\| \leqslant \sqrt{2}\|\xi\|_{\bar{B}}$. The fact that the mapping $\rho_{0}$ given by (2.14) is an algebra homomorphism implies that the same is true for the mapping $\xi \mapsto \breve{T}_{\xi}, H_{0} \rightarrow \mathscr{B}\left(E_{\mathrm{R}}\right)$, and hence

$$
\rho: \quad \xi+\lambda 1 \mapsto \check{T}_{\xi}+\lambda I_{E_{\mathrm{R}}}, \quad \widetilde{H}_{0} \rightarrow \mathscr{B}\left(E_{\mathrm{R}}\right),
$$

is a continuous, unital algebra homomorphism, where $I_{E_{\mathrm{R}}}$ denotes the identity operator on $E_{\mathrm{R}}$.

We shall now complete the proof by showing that this homomorphism $\rho$ is a right inverse of $\psi$. Since $\psi$ and $\rho$ are both unital, it suffices to show that $\psi \circ \rho(\xi)=\xi$ for each $\xi \in H_{0}$, which amounts to showing that $(\operatorname{Ad} U) \circ \Theta_{0}\left(\breve{T}_{\xi}\right)=\tau_{\xi}$ by the definitions of $\rho, \psi$, and $\Upsilon$. According to (2.12), both sides of this identity belong to $\mathscr{T}$, so the fact (to be established below) that

$$
T=T^{\prime} \quad \Longleftrightarrow \quad T b_{0}=T^{\prime} b_{0} \quad\left(T, T^{\prime} \in \mathscr{T}\right)
$$

means that it is enough to verify that $\left((\operatorname{Ad} U) \circ \Theta_{0}\left(\breve{T}_{\xi}\right)\right) b_{0}=\tau_{\xi}\left(b_{0}\right)$, or, equivalently, that

$$
\Theta_{0}\left(\breve{T}_{\xi}\right) U b_{0}=U \xi
$$

To prove (2.17), let $T=\tau_{\eta}+\lambda I_{H} \in \mathscr{T}$ be given, where $\eta \in H_{0}$ and $\lambda \in \mathbb{K}$. Then we have $T b_{0}=\eta+\lambda b_{0}$, which uniquely determines both $\eta$ and $\lambda$, and hence $T$, because $b_{0}$ is orthogonal to $H_{0} \ni \eta$. This establishes the implication $\Leftarrow$, while the converse is clear.

We begin our proof of (2.18) by observing that $\sigma_{m}=\sum_{j=1}^{m} e_{j}$ is a unit vector in $J B_{i}$ for each $m \in \mathbb{N}$ and $i \in \mathbb{N}_{0}$, and hence $\left(\sigma_{m} \otimes \eta\right)_{m \in \mathbb{N}}$ is a norm-bounded sequence in $Y \subseteq Y^{* *}$ for each $\eta=\sum_{i=0}^{\infty} \eta_{i} b_{i} \in \bar{B}$. Since the elements of the form $e_{n}^{*} \otimes \zeta=\left(\zeta_{i} e_{n}^{*}\right)_{i \in \mathbb{N}_{0}}$, where $n \in \mathbb{N}$ and $\zeta=\sum_{i=0}^{\infty} \zeta_{i} b_{i} \in \bar{B}$, span a norm-dense subspace of $Y^{*}$, and

$$
\left\langle\sigma_{m} \otimes \eta, e_{n}^{*} \otimes \zeta\right\rangle=\sum_{i=0}^{\infty} \eta_{i} \zeta_{i}=\left\langle e_{n}^{*} \otimes \zeta, \Phi \otimes \eta\right\rangle \quad(m \geqslant n)
$$


we conclude that the sequence $\left(\sigma_{m} \otimes \eta\right)_{m \in \mathbb{N}}$ weak $^{*}$-converges to $\Phi \otimes \eta$ in $Y^{* *}$ by a standard elementary result (see, e.g., [3, Exercise 3.3]), as noted by Read [7, p. 315].

Writing $\xi$ as $\xi=\sum_{n=1}^{\infty} \xi_{n}\left(x_{n}+y_{n}\right)$, we obtain

$$
\begin{aligned}
Q_{N} T_{\xi}\left(\sigma_{m} \otimes b_{0}\right) & =Q_{N}\left(\sum_{n=1}^{\infty} \xi_{n} \sigma_{m} \otimes \beta_{n}\right) & \text { by }(2.13) \\
& =\sum_{n=1}^{\infty} \xi_{n} Q_{N}\left(\sigma_{m} \otimes \beta_{n}\right)=\sum_{n=1}^{\infty} \xi_{n} Q_{N}\left(\sigma_{m} \otimes\left(x_{n}+y_{n}\right)\right) & \text { by }(2.6)-(2.7) \\
& =Q_{N}\left(\sigma_{m} \otimes \sum_{n=1}^{\infty} \xi_{n}\left(x_{n}+y_{n}\right)\right)=Q_{N}\left(\sigma_{m} \otimes \xi\right) &
\end{aligned}
$$

for each $m \in \mathbb{N}$. This implies that

$$
Q_{N}^{* *} T_{\xi}^{* *}\left(\Phi \otimes b_{0}\right)=\mathrm{w}_{m \rightarrow \infty}^{*} \lim _{m} Q_{N} T_{\xi}\left(\sigma_{m} \otimes b_{0}\right)=\mathrm{w}_{m \rightarrow \infty}^{*}-\lim _{N} Q_{N}\left(\sigma_{m} \otimes \xi\right)=Q_{N}^{* *}(\Phi \otimes \xi)
$$

because the bidual of an operator $T$ is a weak ${ }^{*}$-continuous extension of $T$. Combining this identity with the diagrams (2.11), (2.1), and (2.16), we can now verify (2.18):

$$
\begin{aligned}
\Theta_{0}\left(\breve{T}_{\xi}\right) U b_{0} & =\Theta_{0}\left(\breve{T}_{\xi}\right) \pi_{E_{\mathrm{R}}} Q_{N}^{* *}\left(\Phi \otimes b_{0}\right)=\pi_{E_{\mathrm{R}}} \breve{T}_{\xi}^{* *} Q_{N}^{* *}\left(\Phi \otimes b_{0}\right) \\
& =\pi_{E_{\mathrm{R}}} Q_{N}^{* *} T_{\xi}^{* *}\left(\Phi \otimes b_{0}\right)=\pi_{E_{\mathrm{R}}} Q_{N}^{* *}(\Phi \otimes \xi)=U \xi,
\end{aligned}
$$

which completes the proof.

Acknowledgements. We are grateful to Garth Dales (Lancaster) and Zinaida Lykova (Newcastle) for having drawn our attention to the questions which motivated this paper, that is, whether every extension of $\mathscr{B}(E)$ which splits algebraically also splits strongly, and whether $\mathscr{B}(E)$ may have homological bidimension at least two. We would also like to thank Graham Jameson (Lancaster) and to acknowledge several helpful conversations with Charles Read (Leeds).

\section{REFERENCES}

1. W. G. Bade, H. G. Dales, and Z. A. Lykova, Algebraic and strong splittings of extensions of Banach algebras, Mem. Amer. Math. Soc. 137 (1999).

2. H. G. Dales, Banach algebras and automatic continuity, London Math. Soc. Monogr. Ser. 24, Clarendon Press, Oxford, 2000.

3. M. Fabian, P. Habala, P. Hájek, V. Montesinos Santalucía, J. Pelant, and V. Zizler, Functional analysis and infinite-dimensional geometry, CMS Books in Mathematics/Ouvrages de Mathématiques de la SMC 8, Springer-Verlag, New York, 2001.

4. B. E. Johnson, Continuity of homomorphisms of algebras of operators, J. London Math. Soc. 42 (1967), $537-541$.

5. T. Kania, N. J. Laustsen, and R. Skillicorn, A singular, admissible extension which splits algebraically, but not strongly, of the algebra of bounded operators on a Banach space, J. Funct. Anal., to appear; available online at arXiv: 1603.04275

6. N. J. Laustsen and R. Skillicorn, Splittings of extensions and homological bidimension of the algebra of bounded operators on a Banach space, C. R. Math. Acad. Sci. Paris 354 (2016), 459-463.

7. C. J. Read, Discontinuous derivations on the algebra of bounded operators on a Banach space, J. London Math. Soc. 40 (1989), 305-326. 
8. R. Skillicorn, Discontinuous homomorphisms from Banach algebras of operators, Ph.D. Thesis, Lancaster University, 2016; available online at

http://www . research.lancs.ac.uk/portal/en/publications/discontinuous-homomorphismsfrom-banach-algebras-of-operators (09187dbe-6653-4979-a132-3f65d393bf c5)/export.html

Department of Mathematics and Statistics, Fylde College, Lancaster University, LanCASTER LA1 4YF, UNITED KINGDOM

E-mail address: n.laustsen@lancaster.ac.uk

Department of Mathematics and Statistics, Fylde College, Lancaster University, LanCASTER LA1 4YF, UniTED KingDom

E-mail address: r.skillicorn@lancaster.ac.uk 